AperTO - Archivio Istituzionale Open Access dell'Università di Torino

\title{
Digital heritage and avatars of stories
}

\section{This is the author's manuscript}

Original Citation:

\section{Availability:}

This version is available http://hdl.handle.net/2318/139817

since 2016-12-01T09:04:52Z

Publisher:

ACM - Association for Computing Machinery

Published version:

DOI:10.1145/2517978.2517991

Terms of use:

Open Access

Anyone can freely access the full text of works made available as "Open Access". Works made available under a Creative Commons license can be used according to the terms and conditions of said license. Use of all other works requires consent of the right holder (author or publisher) if not exempted from copyright protection by the applicable law. 


\section{Digital Heritage and Avatars of Stories}

\author{
Vincenzo Lombardo \\ CIRMA and Dipartimento di Informatica \\ University of Torino \\ Torino, Italy \\ vincenzo.lombardo@unito.it
}

\author{
Antonio Pizzo \\ CIRMA and Dipartimento di Studi Umanistici \\ University of Torino \\ Torino, Italy \\ antonio.pizzo@unito.it
}

\begin{abstract}
A relevant issue in the annotation of digital heritage is the abstraction of concepts in a cross-media context. This is true in the case of dramatic media (e.g., screenplay, performance, TV series, video-game, feature film), where the performance has the same relevance of the text, and can be read as a text itself. The primary notion underpinning these two types of texts is the story, with its dramatic qualities shared among the different texts. Thus, following Ryan's definition of "avatar of story", each format can be seen as an avatar of this primary notion This paper presents a system for the collaborative annotation of the story elements in media heritage. Story elements are employed to describe the dramatic qualities (e.g., story agents, agents' goals, conflicts of agents), abstracting from the specific media in which they appear. The system consists of an ontology-based schema and a web platform for the annotation of the dramatic metadata on the digital heritage items (in textual or audiovisual form). The paper also describes a module for the visualization and exploration of such metadata, for crosscomparisons in drama studies. The system was tested on the comparison between the agent's intentions in the story representation and the incidents appearing in the timelines of the specific performances.
\end{abstract}

\section{Categories and Subject Descriptors}

H.5 [Information Interfaces and Presentation]: User Interfaces; J.5 [Computer Applications]: Arts and $\mathrm{Hu}-$ manities-Arts, fine and performing

\section{General Terms}

Documentation, Human Factors, Standardization, Languages.

\section{Keywords}

dramatic media, ontological representation, information visualization

Permission to make digital or hard copies of all or part of this work for personal or classroom use is granted without fee provided that copies are not made or distributed for profit or commercial advantage and that copies bear this notice and the full citation on the first page. Copyrights for components of this work owned by others than the author(s) must be honored. Abstracting with credit is permitted. To copy otherwise, or republish, to post on servers or to redistribute to lists, requires prior specific permission and/or a fee. Request permissions from Permissions@acm.org.

DH-case '13 September 10 2013, Florence, Italy

Copyright is held by the owner/author(s). Publication rights licensed to ACM. ACM 978-1-4503-2199-0/13/09 ...\$15.00

http://dx.doi.org/10.1145/2517978.2517991.

\section{INTRODUCTION}

In recent years, the efforts of digitization of Cultural Heritage are providing common users with access to large amount of materials (see, e.g., Europeana ${ }^{1}$ ). However, the amount of metadata is very restricted, items come with very short descriptions and lack contextual information. Complying with the UNESCO Convention for the Safeguarding of Intangible Cultural Heritage, in this paper we address the "cultural" side of the digital heritage, thus pointing to the social and symbolic values [33]. In particular, we address the notion of story, working on the annotation of narrative heritage items. In her book, M.L. Ryan says that

narrative is a cognitive construct with an invariant nucleus of meaning, but this construct can take a variety of shapes, which we may call avatar of story, and it can be actualized to variable degrees, depending on how many of its core conditions are fulfilled [31, p. xviii].

Thus, the annotation of a text can be improved if we also take into account this primary notion of story that underpins it. This can be particularly useful when we deal with a type of narrative text such as drama. In fact, drama is a text that works as an input for another type of text, the performance. Before the drama becomes the performed action on stage (or on screen), it needs to be re-scripted into the specific format for the mise-en-scène. For example, very rarely the whole original Hamlet text is performed as it is (leaving aside language translations), it is normally re-scripted (usually shortened) to better fit the constraints of the performance. Then it is re-coded into speeches, actions, stage designs and layouts, etc. Staging, either for theatre, cinema, or TV, before occurring, always requires this sort of interpretation. In the case of the dramatic media [14], if the annotation has to be effective in encoding the content, it must include the primary elements of the story, because those elements will be the ones that build the cognitive representation of the story that we know as Hamlet, within the overall perception of the audience, who is attending one specific avatar. In the words of Elam, who formulates the abstract notion of story as fabula:

It is clear that the fabula, being an abstraction from the sujzet/plot as such, is a paraphrase of a pseudo-narrative kind, made, for example, by a spectator or the critic in recounting the 'story' of the drama. It is usually the prime object of

\footnotetext{
${ }^{1}$ http://www.europeana.eu/
} 
the spectator's hypothesizing in witnessing the representation [12, p. 120].

The variations over the numerous versions of the story can be considered quite common in the wide domain of the intangible cultural heritage, as in traditional folk fable where there is a flourishing of versions (for example the story of Cinderella from the oral tale to Perrault's or Disney's versions).

In theatre, the representation can be considered a sort of translation that converts the information provided by the written text into the performance, transcoding the "dramatic text" into the "performance text" [22]. In a drama, the most important aspects of the translation are the dramatic features of the text (e.g. characters, intentions, conflicts, emotions). Those, rather then the actual words used or the original language of the author, can be truly played in different forms no matter of the media used (a live performance, a movie, a tv serial, etc.). These features can be considered as the intangible heritage of a dramatic item. that:

Thus we can conclude, with Pfister and Halliday's words,

a number of different texts can be based on one and the same story and also that the same story may even be presented as texts in different media [28, p. 197].

Along with the preservation of the data of the actual narrative text (be it a movie, a movie script, a recorded performance, a play, etc.), and the physical storage of the data, in analogical and digital formats, we claim that metadata must encode the abstract notion of "story" (together with the specific elements that constitute a story) as further information to be maintained. The notion of "story" is widely acknowledged as the construction of an incident sequence [4], that, abstracting from the mise-en-scène properties, is motivated by the cause-effect chain [30]; this chain results from a complex interplay among agents, events, and environments, well known in playwriting techniques [11]. In this paper, we propose to annotate the items of the dramatic media with metadata about the notion of "story". Indeed, though there are a number of examples where the physical support (film and tape) has been augmented with metadata in order to preserve/represent those information, yet there is not a shared system to represent the symbolic features, such as the "story".

In the last decade, there have been a number of approaches for enriching media and heritage items with metadata. Some authors have resorted to Wikipedia, which offers in-depth descriptions and links to related articles, and is thus a natural target for the automatic enrichment of heritage items (see, e.g., [1]). In the field of media indexing, and the specific domain on performing arts, MyStoryPlayer is a recent on purpose designed interface for semantic annotation of documents (such as video, audio, text, image). MyStoryPlayer uses RDF to allow the users in the community to annotate a multimedia repository and to navigate in the annotations creating its own non-linear experience/path [3]. Finally, we can consider a relevant source of information the user-generated metadata, such as the tags that are freely inserted by users to annotate the items contained in in public video repositories (e.g., YouTube), although in most cases these annotations concern the resource (such as, e.g., Title, Actor, Director) and in very few cases the content [21].
Because the fruition of dramatic media mostly focuses on enjoying the story rather than appreciating the aesthetic features (the latter are appraised by professionals and knowledgeable users), we propose a suite for the annotation, based on the semantic web technologies, relying on a user tagging driven by a narration model that is encoded in an ontology, and an access that takes advantage of a visualization tool that reveals relevant dramatic properties of the item. Hence, the application we present is designed to model, annotate, and visualize the dramatic values of the dramatic media, and provides a cross-media, abstract representation of a narration sequence (a timeline of incidents) and of the complex interplay.

\section{ANNOTATION AND VISUALIZATION OF STORIES}

In this section we present the CADMOS suite, a set of applications for the modeling, annotation and visualization of the story elements in a dramatic media object (CADMOS is an acronym for Character-centred Annotation of Dramatic Media ObjectS ${ }^{2}$ ). Given an audiovisual item, the annotator, being her/him a scholar or an enthusiast, splits the item into units (Segmentation), and defines a timeline of incidents as perceived from the movie. Units are independently identified through the boundaries of the actions, and then incidents are identified internally. Then, he/she annotates the metadata for each unit, encoding the character's intentional behaviors in terms of goal, plans and achievement states, also with the support of the information from the original text (Annotation phase). Timeline incidents, actions, goals, and plans are encoded according to the Drammar ontology (see below). Following, the timeline incidents and the plan descriptions are mapped thanks to the actions that are present in both representations. The result is a comparison between the timeline of incidents featured into the movie with the character's intentions into the original text. Finally, the encoding is displayed by matching the timeline incidents with the actions and plans assigned to the characters, to reveal the structure of the story plot in a visualization (see below). The visualized structure features an augmented timeline at the top (with states holding between units made explicit after plan/incident mapping) and agents' plans, hierarchically organized, aligned with incidents.

Now we introduce the ontology, the web-based application for annotating the metadata, and the visualization tool. The reported examples are related to the "nunnery scene" of Hamlet, the film directed by Laurence Olivier (Two Cities Film production, UK, 1948), based on Shakespeare's text.

\subsection{Ontology Drammar}

The story elements are encoded in an ontology (see [17] for an introduction), encoded in the OWL language. Ontology Drammar $^{3}$ is designed with the twofold goal of providing an instrument for the conceptual modeling of story facts, and a formal tool for the devise of an annotation schema for building the metadata of a narrative document.

There exist other approaches for the formal encoding of the story elements. The Story Intention Graph[13], similarly to Drammar, relies on the representation of agents' intentions to build an interpretive layer of a narrative text;

\footnotetext{
${ }^{2}$ http://cadmos.di.unito.it

${ }^{3}$ For a more detailed account see $[7]$
} 


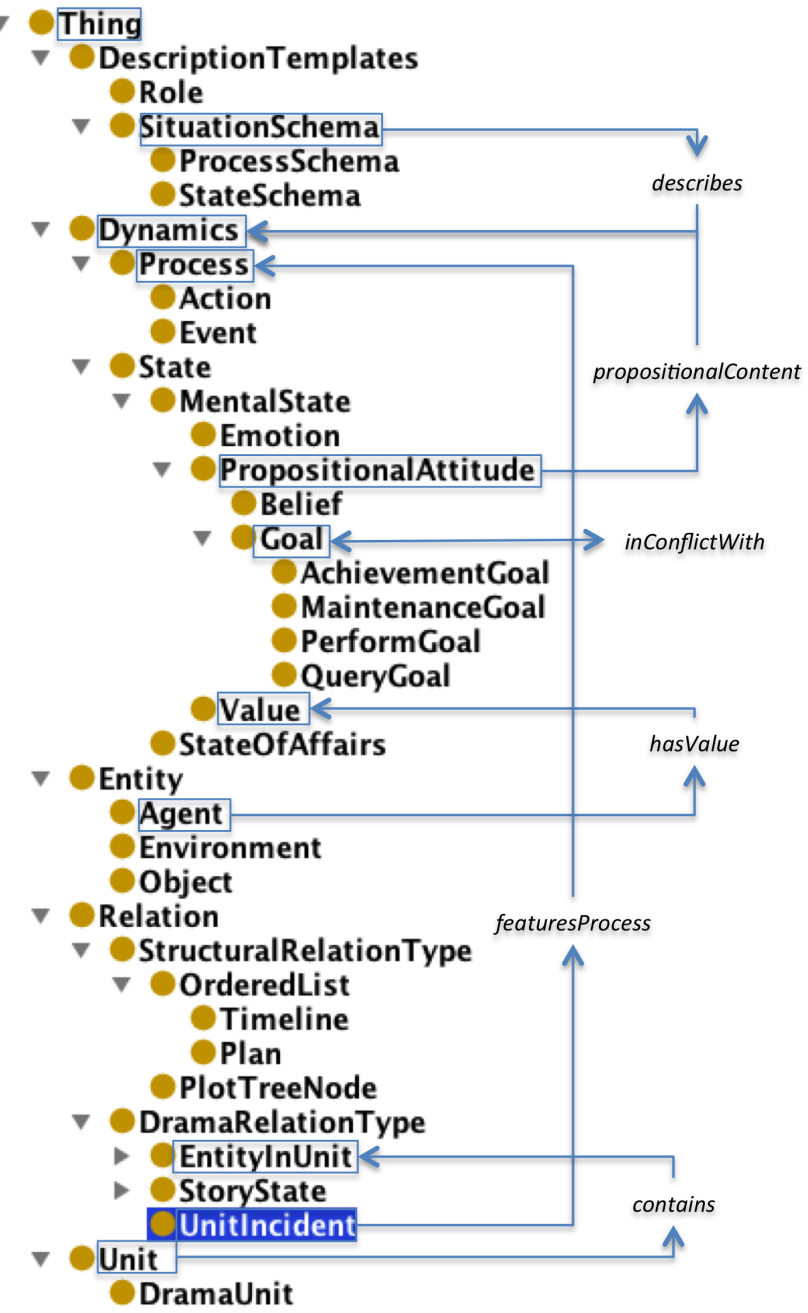

Figure 1: Excerpt of the Drammar ontology, with some classes and relations.

however, the resulting structure is flat and does not account for the motivations originating from the long term goals of the agents and spanning large portions of text; contrarily to Drammar, it does not rely on a formal ontology. The Stories ontology $^{4}$, developed in collaboration with the BBC for the application in news, the storylines of Doctor Who episodes, and historical facts, is mostly event-(instead of character) based and has no interpretive intents, being limited to a timeline.

The top level of Drammar consists of five disjoint classes: Entity, Relation, DescriptionTemplates, Dynamics, and Unit (see fig.1).

A unit (Unit class) is a chunk of the story, identified by the annotator according to actional boundaries, i.e. it is characterized by the occurrence of some incidents, of which we can recognize beginning and end.

Entity models all the story elements participating in the unit, namely Agent (the characters that intentionally act in the incidents), Object (the objects that do not own intentions), and Environment (the locations where the incidents take place). All such entities feature qualities (e.g. speed or color), status (e.g., open/closed), a type (e.g., an indi-

\footnotetext{
${ }^{4}$ http://www.contextus.net/stories
}

vidual telephone Object in a scene belongs to the class of the telephones), a message conveyed by an object (e.g., a billboard with the name of a toothpaste brand). In general, following the paradigm of linked data[18], all the qualities are represented as URI's referring to some external commonsense or specific ontology. Drammar refers to two external large-scale semantic resources for the description of the commonsense knowledge, namely the two ontologies Suggested Upper Merged Ontology (SUMO, [26]) and Yet Another Great Ontology (YAGO [34]), merged into YAGO-SUMO [9], which provide very detailed information about millions of situations, including entities (agents and objects), processes/actions, and events. Terms in YAGO-SUMO are accessed through a lexical resource, the WordNet lexical data base $[23]^{5}$; in particular, we have realized an interface for supporting the manual selection of meaning, extending the vocabulary to a multilingual setting (through the lexical data base MultiWordNet [29]), to increase the interoperability of the annotation data across languages. In some particular cases, some features refer to some specific data (e.g., the string "Where is your father?" to represent the message conveyed by Hamlet's utterance to test Ophelia's loyalty).

The story evolves through a sequence of incidents, that are possible because the story world is in some state. The class Dynamics models incidents and states through the subclasses Process (in turn, intentional Action or unintentional Event), and State. Entities participate in processes and states according to some Role, that is constrained by a SituationSchema (split into ProcessSchema and StateSchema). All the schemata belong to the class DescriptionTemplate, which provide the templates for a number of ontological structures, sometimes referring to a pattern (see below for incidents). Templates are described by the frames of Framenet [2], describing situations, processes/actions, and/or events through a semantic template that depicts the situation in terms of roles played by the elements which participate in it. The class State is decomposed into several subclasses: first, we must distinguish between StateOfAffairs, i.e. states that hold in the story world, and Mentalstate, i.e. states that are claimed to hold within the mind of one Agent. Mental states are at the core of the intentional behavior of agents.

Agents, in fact, are the most complex entities. Inspired by the BDI theory [5] [25] [27], agent mental states concern one of the following classes:

- Belief: the agent's subjective view of the world (e.g., Hamlet believes "Ophelia is not loyal");

- Emotion: what the agent feels (e.g., Hamlet is in "Love" with Ophelia);

- Value: the moral qualities of an agent, that are put at stake along the narrated story (e.g., Hamlet's value put at stake is Sincerity);

- Goal: formulated by the agents after a deliberative process that takes into account beliefs, emotions and values at stake; in accordance with agent theories, Drammar acknowledges different goal types (cf. [35]), depending upon their propositional content: Perform goal, that aims at the execution of a certain action; Achievement goal, that aims at the achievement of

\footnotetext{
${ }^{5}$ See the portal http://www.ontologyportal.org/
} 
a certain state of the world; Query goal, a type of achievement goal, where the state to be achieved is a belief state of the agent itself; Maintenance goal, that aims at keeping or restoring a certain state in the story world.

In the nunnery scene, the Ophelia's goal of letting Hamlet reveal his feelings is an achievement goal, while the Hamlet's denying of have ever given anything is a perform goal.

In the nunnery scene, in Olivier's Hamlet, Ophelia (Agent) is waiting for Hamlet (Agent) because she wants (Goal) that he reveals his inner feelings. When he arrives she tries to returns (Action) some love gifts (Entity). In the scene Ophelia feels a distress (Emotion) because the authority (Value) of her father is at stake. Hamlet refuses the gift and denies he ever gave her anything.

The class Relation is the core of the ontology, because it relates the entities and the processes/states to the units (class DramaRelationType) and the relations over units (class StructuralRelationType). The class DramaRelationType defines the relationships between agents and mental states in a specific Unit. The class AgentInUnit (a subclass of EntityInUnit) permits to associate an instance of an Agent to a pursued Goal. Similarly, each unit is also associated with a set of actions (performed by the agent) in order to achieve the goal. The property hasAction connects the AgentInUnit class with the actions the agent performs and the hasGoal property connects each action with an agent's goal in a unit. The actions occurring in a unit are motivated by (at least) one agent's goal (the class EntityInUnit contains all the classes that describe the behavior of the agent specific in a unit). and unit boundaries coincide with the achievement or failure of some agent's goal. For example, the unit where Ophelia returns the gifts to Hamlet, ends when she finish the action of lending them. A unit is a dramatic (DramaUnit) when a goal is in conflict with some other goal (either of the same character or of another agent) or with an event (see fig. 2). The units that do not have conflicts but participate to the the construction of a dramatic unit are defined as actional and contains only agent's actions. We carefully distinguish among those that have a dramatic worth from the others, which are instrumental and simply do not have any dramatic implication on the story. To model the conflict between goals, the inConflictWith property links a character's goal in a certain unit with another character's goal, as in fig. 2. The property can link also goals form different units and of the same character too (the latter being the case of an inner conflict). For each drama unit, we can have sub drama units (belonging to DramaUnit class) and/or simple actional units (belonging to Unit class). For each actional unit we can have only sub actional unit.

The class StructuralRelationType models the structural relations existing over the units (inter-unit relations) and the relations among the entities into the unit (intra-unit relations). The first are modeled as a plot tree by recursively linking the units as a tree structure (class PlotTreeNode). The second, class StructuralRelationType, accounts for the ordered lists Timeline and Plan. Timelines are made of incidents; plans consist of actions and precondition and effect states, which precede and follow the actions, respectively; recursively, plans are made of sub-plans and precondition/effect states. This means that the plans represent the abstract cognitive deliberation of the agent, but when they are instantiate into the timeline, they are made of state of affairs, actions, i.e. only the thing they are truly happened are shown.

\subsection{CADMOS Annotation and Visualization}

Within project Cadmos we have developed a web based annotation tool, that has been designed to carry out the encoding of the annotation in formal languages transparent to the annotator. The annotation process starts by identifying the meaningful units of the item, by marking its boundaries through a video player interface; then, selecting the appropriate tabs, the annotator introduces the metadata for the story elements (agents, objects, environments); finally, the annotator retrieves the incident templates (a similar template for actions, events, and states), that are filled with the story elements identified above in appropriate roles. The annotation of the incidents is conducted by filling a set of templates that describe the narrative elements of a unit; the appropriate templates are identified through natural language terms that are used to retrieve the template schemata in the lexical and commonsense knowledge resources.

As an example of annotation, we describe the annotation of a story incident (see Figure 2), driven by the Time Indexed Situation design pattern developed in the descriptive ontology DOLCE [16]. The incident is extracted from the "nunnery scene" in the Third Act of Shakespeare's Hamlet. In this scene, Ophelia is sent to Hamlet by Polonius and Claudius to confirm the assumption that his madness is caused by his rejected love. According to the two conspirers, Ophelia should induce him to talk about his inner feelings. In the middle of the scene Hamlet puts Ophelia on a test to verify her loyalty. Because he guesses (correctly) that the two conspirers are hidden behind the curtain, he asks the girl to reveal where her father Polonius is. She decides to lie and replies that he is at home. Going from top to bottom in the figure, the unit contains two incidents. The incident on the left is a process triggered by Hamlet's achievement goal to confess about her arrangement with Claudius and her father Polonius. The goal is described a schema that is retrieved through the term "confess" and access the SUMO concept "Stating" and the generic frame provided by Verbnet (this occurs because this verb has no frame mapped in Framenet), with two roles, Agent and Patient, filled by Hamlet and the arrangement respectively. The process that tries to achieve this goal (actually failing) is a testing executed by Hamlet (the Speaker role of the Frame Questioning in Framenet) on Ophelia (the Addressee) about the Topic "Polonius' location" through the Message "Where is your father?". The incident on the right achieves a Perform goal (in which the goal corresponds to the success of an action) of lying, carried on by Ophelia, who is the Speaker of a Prevarication frame (in Framenet), with Hamlet as Addressee and the "Polonius' location" as Topic. The two goals are triggered by the two values that are put at stake in this unit: for Hamlet is sincerity to be put at stake, for Ophelia the authority of her father.

Again from the "nunnery scene", we can analyze the plan Ophelia uses to provoke the prince, by giving back the gift once received as a love sign. The plan is: $P_{b 1}^{O p h e l i a}[A G$ : Ophelia wants provoke Hamlet $]=$

SOA(True) : Ophelia neglected

$A$ : Ophelia returning Gift to(Hamlet)

SOA(False) : Hamlet has Gift

In this plan, Ophelia, who has the Achievement Goal of 


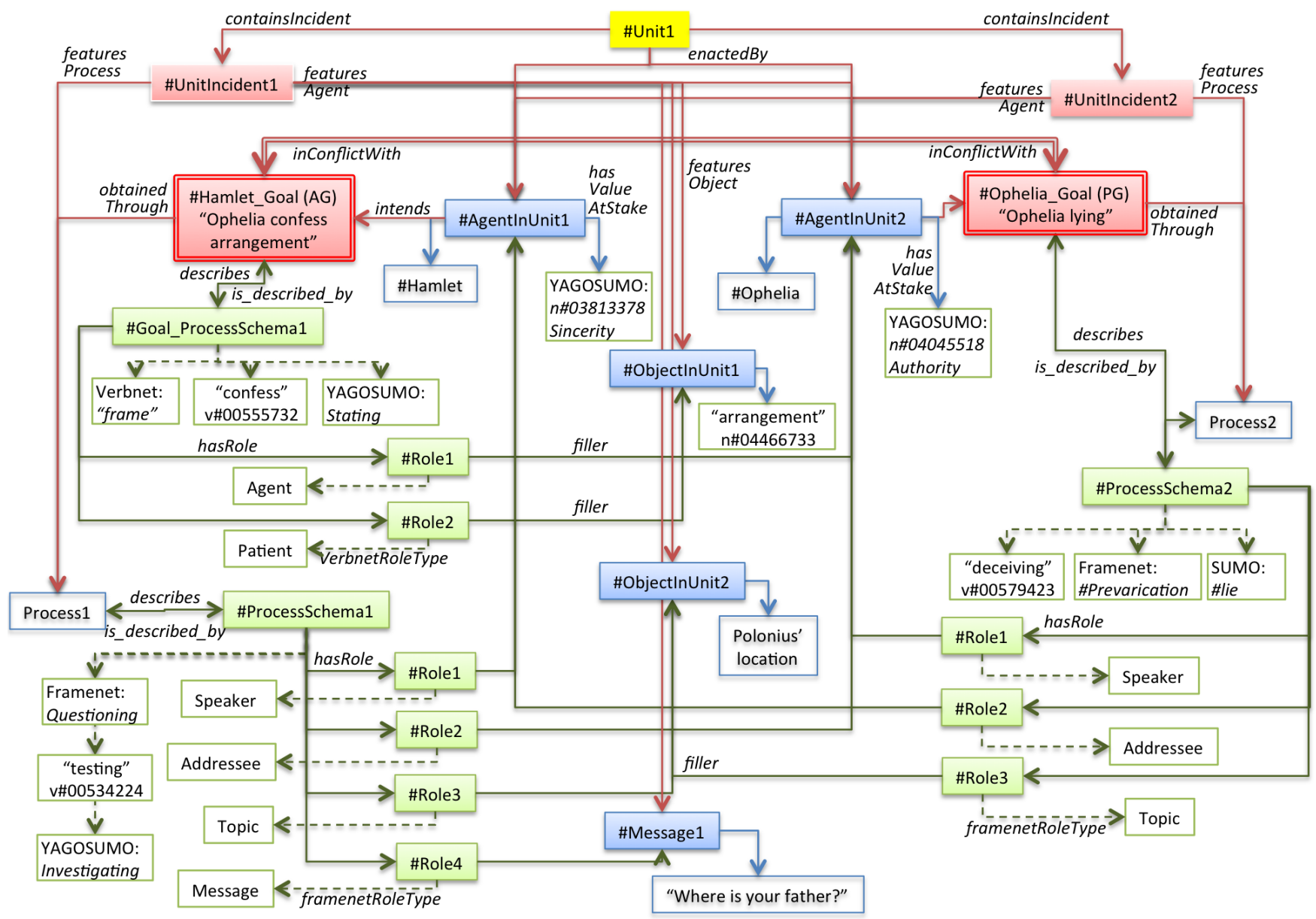

Figure 2: The annotation of the Unit \#Unit1 where the Agent (\#Hamlet tests (concept \#Investigating, frame \#Questioning) the Agent \#Ophelia's sincerity about her arrangement with Claudius and her father Polonius (\#UnitIncident1) and she lies (concept \#lie, frame \#Prevarication) about it. The structure is built upon relations on roles and role fillers. Testing and lying are the two processes that describe the two incidents, respectively. Notice the conflict between the Hamlet's goal (the Achievement Goal of making Ophelia confess the arrangement) and Ophelia's Performing Goal to lie.

provoking Hamlet, since the precondition state of affairs of "Ophelia being neglected" holds, the action to return the Gift to Hamlet takes place, with the effect state of Hamlet having the Gift (this state of affairs will result false because of a successful conflict goal of Hamlet to reject the Gift).

In [21] we present the validation of the platform through a preliminary test with annotators. Currently, we have the following contents in our repository. The complete feature film North by Northwest, a 2:30 min animation short, a 3:00 min music videoclip, a $30 \mathrm{sec}$ advertisement, an original 12 min movie, and 10 scenes from famous feature films.

\subsection{Mapping and visualization}

Both timeline and plan modeling relies on the generic class OrderedList, that represent the incidents' positions on an ordered list. An instance of Process or State refers to some position in the Timeline or in a Plan, respectively. Following, the reasoner infers that some ordered list of incidents in the timeline belongs to some plan. (actually, it can happen that some incident is mapped onto more than one plan in the case of equivocals). The reasoner works with inferences of an ontological nature and with a SWRL IF-THEN rule that validates the mapping of some incident to some plan action. Then, the timeline of incidents is augmented through an off-line algorithm that takes as inputs the timeline and the plans, and returns as output an OrderedList that contains the incidents of the Timeline, in the same partial order as in the Timeline, interspersed with precondition and effect states (agglomerated into story world states). So, as in fig. 3 , if a (plan)state $S_{1}$ is a precondition of the action $A_{1}$ in the plan $P_{B 1}^{Y}$, and the action $A_{1}$ is mapped on the incident $I_{2}$ in the Timeline, then a state $S_{2}$, that is the same as $S_{1}$ is inserted in the Timeline before $I_{2}$ (and after the preceding incident in the Timeline). The augmented timeline OrderedList features a total order over incidents and states.

In the example of the "nunnery scene" and the plan $P_{b 1}^{\text {Ophelia }}$, the timeline contains the actional incident I_OLI0016 (Ophelia returning gift to Hamlet), which is mapped onto A0005 (Ophelia returning gift to Hamlet). A plan participates to the mapping and the augmentation of a timeline when the order 


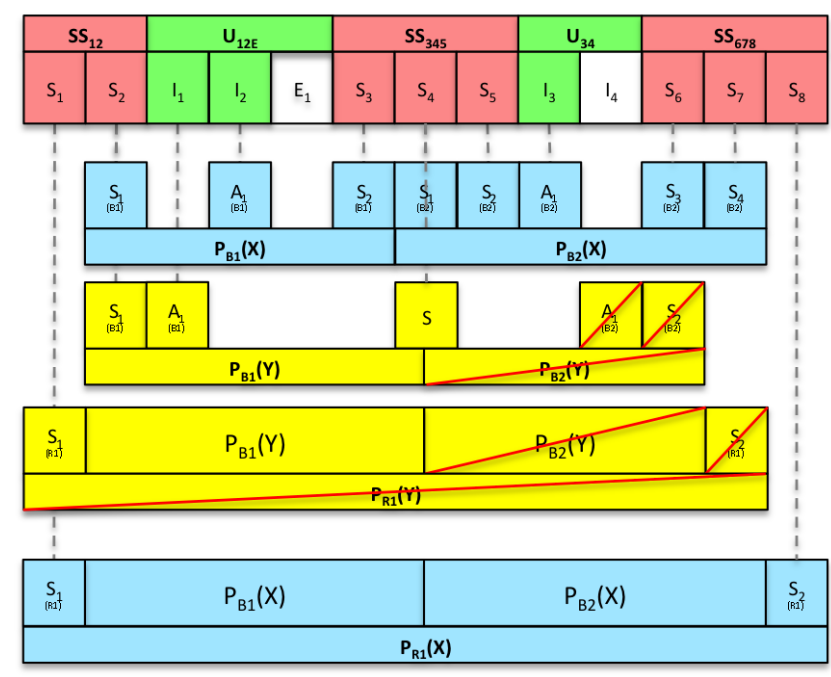

Figure 3: General schema of the visualization: augmented timeline, made of units (made of incidents) and story states (made of states projected from plans), at the top; agents' plans, made of actions and states aligned with timeline incidents and states respectively, at the bottom.

of the incidents on the timeline respects the order of the mapped actions in the plan. In our example, since we have the mapping $\mathrm{I}_{-} \mathrm{OLI}_{0016}-\mathrm{A}_{0005}$, and the subsequent mapping I_OLI ${ }_{0017}$ (Hamlet denying gift)- $\mathrm{A}_{1112}$ (Hamlet refuse gift), the plan $\mathrm{P}_{P_{-} 0003}^{\text {Ophelia }}$ can participate to mapping (though the last part of the plan is not mapped then). Then, we augment the timeline with the states that hold between adjacent incidents on the timeline. States are taken from the preconditions and the effects that are associated with the actions in the plans.

The visualization concerns characters' intentions (or plans), arranged hierarchically on multiple trees that span a unique timeline of events. The abstract structure of visualization is the following. In the top row there is the Augmented Timeline, split into units (U) and story world states (SS). Units are made of incidents, which can be either intentional actions (I), so mapped to actions in agents' plans, or events (E). Story states are collections of single states, which are retrieved from the agents' plans and projected onto the timeline, to form the Augmented Timeline. Events and unmapped incidents are colored in white (e.g. $E$ in the second unit in fig. 3 ). In the lower part of the figure we visualize the plans of the agents, arranged hierarchically. The subscript B means Base, while the subscript $\mathrm{R}$ means Recursive. $X$ e $Y$ are the agents that commit to the plans; $\mathrm{S}$ is a state and $\mathrm{A}$ is an action. In case an action or a state is not mapped, it means that the action or the plan executions failed or the state is not achieved and is false. The latter are represented as barred in the diagram. In figure 4 there is the visualization of the excerpt of "nunnery scene" described above.

\section{DISCUSSION}

In order to validate our approach, we tested the differences between the abstract story derived from the Shakespeare's text and one of its "avatar", namely the specific mise-en-scène in Olivier's movie. The annotation is useful to put upfront the shared elements: in order to represent the abstract story, we encoded both the incidents of both the text timeline and Olivier's movie timeline, and the plans that generate them. The mapping between the plan actions and the incidents of the timeline allows to produce the augmented timeline and to appreciate the differences visually. In Figure 4, top, we show a single timeline that merges the incidents of the text and the movie (of course, most of them coincide): incident origin is identified through a prefix, I_OLI for incidents belonging to the movie timeline, I_TXT for incidents belonging to the text timeline. The movie incidents that are not in the text display a white background; the plans abstracted from the text do not match such incidents.

The visualization helps the user (scholar or enthusiast) to measure the degree of equivalence between the intangible abstract story and the concrete medium representation. In the field of theatre studies, one of the most appreciated topics, in fact, is the evaluation of the differences between the original text and one specific mise-en-scène. The mapping of the plans onto the timeline shows the relation between the description of the characters' behaviors and intentions, as implied by the text Hamlet, and the factual representation of those behaviors in the mise-en-scène (i.e. the actors' performed actions). Overall, we note that most parts of the two timelines overlaps, and only in a few occasions we needed to insert the incidents of the text. In fact, most of the incidents present in both (the text and the movie) had a similar content, and has been possible to use only the I_OLI.

Nevertheless, some differences emerge. In our example, the beginning of the "nunnery scene" in the movie shows a high grade of conformity with the text, albeit there is a clear discrepancy regarding the Hamlet's plan $\mathrm{P}_{1111_{1} 1}$ for which we resort to the text (I_TXT0004), as well as in the plan P1114 that can be matched only with I_TXT0017. This means that the movie does not fully represent the content of the drama, i.e. the artist provides a specific personal interpretation of the abstract story called Hamlet, i.e. the Shakespeare's play.

In the case of drama, it is important to measure the preservation of the dramatic features that constitute the backbone of the story. The representation of such features must go beyond the mere philological approach, which considers, e.g., the correspondence of all the specific incidents or of all the specific lines of the text. This would, in fact, undermine the quality of the performance that resides in the special ability of the artist in re-coding the text, and must take into account the core structure of the story. In other words, the artist (Olivier, in our case) can neglect to display all the lines of the play as well as each action described in the text, and, in the same moment, he can comply with the drama. For example, Olivier's rendering of Hamlet's character seems to add actions that weren't clearly stated in the text. The incident I_OLI0007 (Hamlet exploring the room), I_OLI0008(Ophelia dissimulating), I_OLI0009(Ophelia kneeling), I_OLI 0011 (Hamlet greeting Ophelia), as shown in the white boxes in the visualization (fig. 4), are invented by Olivier. Nevertheless, they may contribute at fulfilling the plan $\mathrm{P}_{1111_{1} 1}$ (Hamlet neglecting Ophelia), as devised in the behaviors of the original text. This depicts a methodology in which the artist, rather than radically transforming the actions written in the text, adds new ones that will enrich the performance. A different case is the plan $\mathrm{P}_{1114}$ (Hamlet explaining moral to Ophelia), which can only be matched 


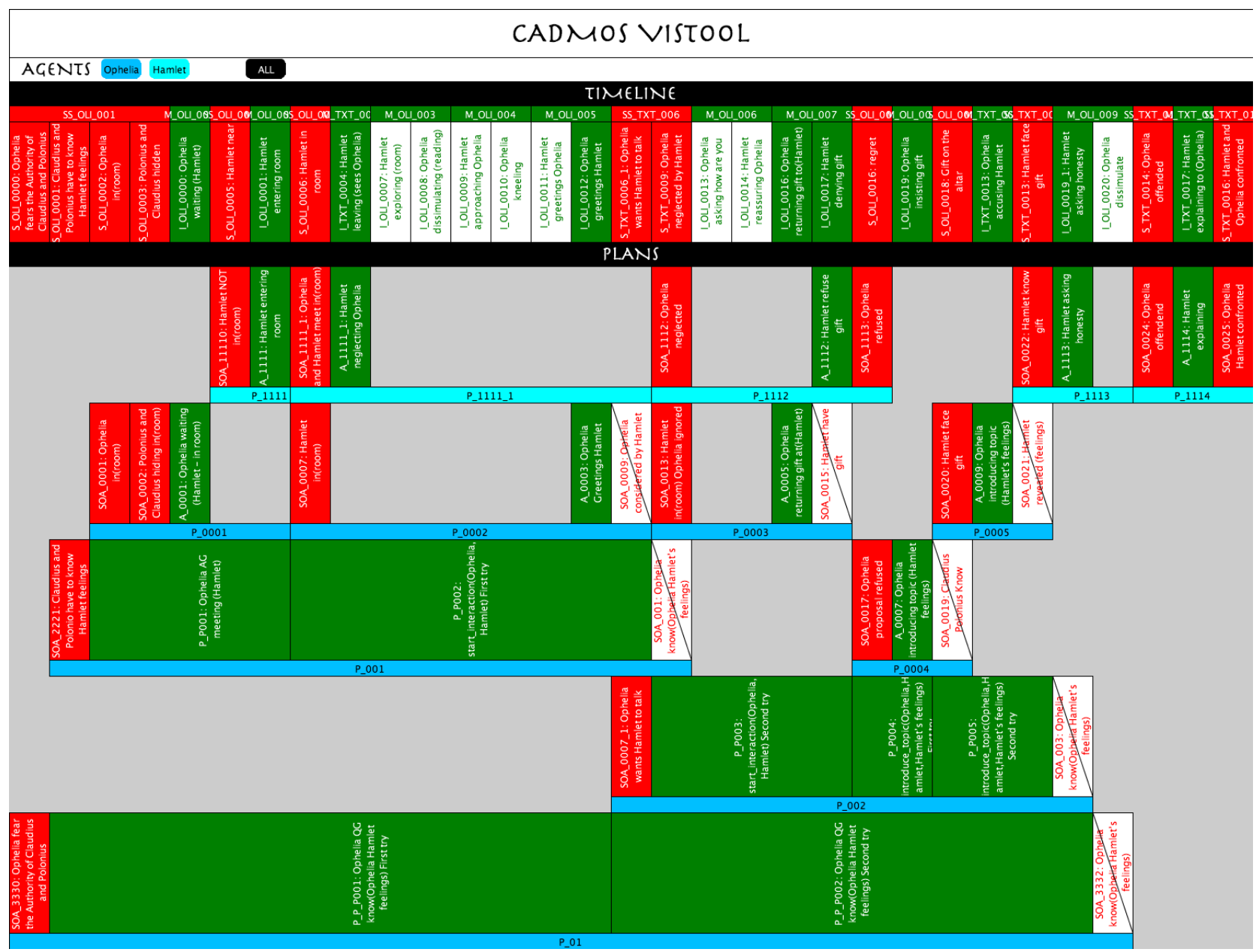

Figure 4: Visualization of an excerpt of Hamlet "nunnery" scene.

with the incidents stated in the text, $\mathrm{I}_{-} \mathrm{TXT}_{0017}$ (Hamlet explaining to Ophelia), because Olivier decided to operate a significative cut, shortening the part in which Hamlet tries to explain the subversion of the moral values in the world of Elsinore's court.

As largely stated in the drama critics (from Diderot till recent scholars [19], [10], [6] [20] [32]), the notion of conflict (represented a relation in the ontology Drammar) plays a key role in shaping the emotional flux of the drama. This has been knows as dramatic arc [15]. The raising of the arc characterizes the drama and is defined by the writer to give the right pace and rhythm to the play. The tension is measured against a configuration of moral values stated in the story [8]. Our visualization adds a new way of detecting this tension arise by measuring the number and the connections of plan failures. In fig. 4, the states of affairs (SOA) forecast in the Ophelia's plans are almost all unrealized (symbolized by the bar in the box). This means that her plans keep on failing and this builds up an emotional tension of the characters. In fact, the character will end the whole scene crying on the floor.

\section{CONCLUSIONS}

In this paper, we have presented a proposal for annotat- ing the metadata of the primary story that is behind the different representations that can be produced, e.g what the audience perceive as a story. Thus we have focused on some selected dramatic features of the narrative items such as character, plan, action, state. Our proposal relies on a computational ontology that encodes the major facts about narration and provides a web-based application for shared annotation. The metadata are displayed through a visualization tool for study and fruition purposes. The approach has been preliminary tested on a study of text/mise-en-scène differences on Hamlet's nunnery scene.

\section{ACKNOWLEDGMENTS}

The work presented here has been partially supported by project CADMOS, funded by Regione Piemonte, Innovation Hub for Multimedia and Digital Creativity, 2010-2012, POR-FESR 07-13.

\section{REFERENCES}

[1] E. Agirre, A. Barrena, O. L. de Lacalle, A. Soroa, S. Fernando, and M. Stevenson. Matching cultural heritage items to wikipedia. In N. C. C. Chair), K. Choukri, T. Declerck, M. U. Doğan, B. Maegaard, J. Mariani, J. Odijk, and S. Piperidis, editors, 
Proceedings of the Eight International Conference on Language Resources and Evaluation (LREC'12), Istanbul, Turkey, may 2012. European Language Resources Association (ELRA).

[2] C. Baker, C. Fillmore, and J. Lowe. The berkeley framenet project. In Proceedings of the 36th Annual Meeting of the Association for Computational Linguistics and 17th International Conference on Computational Linguistics-Volume 1, pages 86-90. Association for Computational Linguistics, 1998.

[3] P. Bellini, P. Nesi, and M. Serena. MyStoryPlayer: Semantic audio Visual Annotation and Navigation Tool. In The 17th International Conference on Distributed Multimedia Systems, Florence, 2011.

[4] D. Bordwell and K. Thompson. Film art : an introduction. McGraw Hill, Boston, 2006.

[5] M. Bratman. Intention, Plans, and Practical Reason. Harvard University Press, Cambridge (MA), 1987.

[6] F. Brunetière. The Law of the Drama. Dramatic Museum of Columbia University, 1914.

[7] M. Cataldi, R. Damiano, V. Lombardo, and A. Pizzo. Lexical mediation for ontology-based annotation of multimedia. In A. Oltramari, P. Vossen, L. Qin, and E. Hovy, editors, New Trends of Research in Ontologies and Lexical Resources, Theory and Applications of Natural Language Processing Series. Springer, 2012.

[8] R. Damiano and V. Lombardo. An Architecture for Directing Value-Driven Artificial Characters. Agents for Games and Simulations II: Trends in Techniques, Concepts and Design, pages 76-90, 2011.

[9] G. De Melo, F. Suchanek, and A. Pease. Integrating yago into the suggested upper merged ontology. In Tools with Artificial Intelligence, 2008. ICTAI'08. 20th IEEE International Conference on, volume 1, pages 190-193. IEEE, 2008.

[10] D. Diderot and L. Crocker. Selected Writings. Classics in the history of thought. Macmillan, 1966.

[11] L. Egri. The Art of Dramatic Writing. Simon and Schuster, New York, 1946.

[12] K. Elam. The Semiotics of Theatre and Drama. Methuen, London and New York, 1980 (1987).

[13] D. K. Elson. Dramabank: Annotating agency in narrative discourse. In Proceedings of the Eighth International Conference on Language Resources and Evaluation (LREC 2012), Istanbul, Turkey, 2012.

[14] M. Esslin. The Field of Drama. Methuen, London, 1988 (1987).

[15] G. Freytag. Technique of the drama, an exposition of dramatic composition and art. S.C. Griggs and Company, Chicago, 1985.

[16] A. Gangemi and V. Presutti. Ontology design patterns. Handbook on Ontologies, pages 221-243, 2009.

[17] N. Guarino, D. Oberle, and S. Staab. What is an ontology? In Handbook on Ontologies. Springer, 2nd edition edition, 2009.

[18] T. Heath and C. Bizer. Linked data: Evolving the web into a global data space. Synthesis Lectures on the Semantic Web: Theory and Technology, pages 1-136, 2011.
[19] G. Hegel. The Philosophy of Art: Being the Second Part of Hegel's Esthetik, in Which Are Unfolded Historically the Three Great Fundamental Phases of the Art-act. BiblioBazaar, 2012.

[20] Y. Lavandier. La dramaturgie. Le clown et l'enfant, Cergy, 1994.

[21] V. Lombardo and R. Damiano. Commonsense knowledge for the collection of ground truth data on semantic descriptors. In Proceedings of the 2012 IEEE International Symposium on Multimedia (ISM 2012), pages 78-83. IEEE Computer Society, 2012.

[22] M. D. Marinis. The Semiotics of Performance. Indiana University Press, 1993.

[23] G. Miller. Wordnet: a lexical database for english. Communications of the ACM, 38(11):39-41, 1995.

[24] M. Naphade, J. R. Smith, J. Tesic, S.-F. Chang, W. Hsu, L. Kennedy, A. Hauptmann, and J. Curtis. Large-scale concept ontology for multimedia. IEEE MultiMedia, 13:86-91, July 2006.

[25] E. Norling and L. Sonenberg. Creating Interactive Characters with BDI Agents. In Proceedings of the Australian Workshop on Interactive Entertainment IE2004, 2004.

[26] A. Pease, I. Niles, and J. Li. The suggested upper merged ontology: A large ontology for the semantic web and its applications. In Working Notes of the AAAI-2002 Workshop on Ontologies and the Semantic Web, volume 28. Edmonton, Canada, 2002.

[27] F. Peinado, M. Cavazza, and D. Pizzi. Revisiting Character-based Affective Storytelling under a Narrative BDI Framework. In Proc. of ICIDIS08, Erfurt, Germany, 2008.

[28] M. Pfister and J. Halliday. The Theory and Analysis of Drama. European Studies in English Literature. Cambridge University Press, 1991.

[29] E. Pianta, L. Bentivogli, and C. Girardi. Multiwordnet: developing an aligned multilingual database. In Proceedings of the First International Conference on Global WordNet, January 2002.

[30] S. Rimmon-Kenan. Narrative Fiction: Contemporary Poetics. Routledge, 1983.

[31] M. Ryan. Avatars of Story. University of Minnesota Press, 2006.

[32] J. Ryngaert and D. Bergez. Introduction à l'analyse du théâtre. Collection Cursus. Série Littérature. Armand Colin, 2008.

[33] L. Smith and N. Akagawa. Intangible Heritage. Key Issues in Cultural Heritage. Taylor \& Francis, 2008.

[34] F. Suchanek, G. Kasneci, and G. Weikum. Yago: a core of semantic knowledge. In Proceedings of the 16th international conference on World Wide Web, pages 697-706. ACM, 2007.

[35] M. van Riemsdijk, M. Dastani, and M. Winikoff. Goals in Agent Systems: A Unifying Framework. In Proceedings of $A A M A S^{\prime} 08,2008$. 